\title{
Re-Analysis of Modeling a Switch from a 13-Valent to 10-Valent Pneumococcal Conjugate Vaccine in Canada: Leveraging Real-World Experience from Belgium
}

\author{
Matt D. Wasserman - Heather L. Sings - Michele R. Wilson • \\ Maarten J. Postma • Marie-Claude Breton • Cheryl McDade • \\ Raymond A. Farkouh
}

Received: September 14, 2018 / Published online: November 20, 2018

(c) The Author(s) 2018

Keywords: Cost-effectiveness analysis; Economic evaluation; Pneumococcal conjugate vaccine; Pneumococcal disease

We recently published an analysis of an infant pneumococcal vaccine policy shift that considered the potential clinical impact and cost-

Enhanced digital features To view enhanced digital features for this article go to https://doi.org/10.6084/ m9.figshare.7297544.

M. D. Wasserman $(\bowtie)$

Pfizer Inc., New York, NY, USA

e-mail: Matt.wasserman@pfizer.com

H. L. Sings · R. A. Farkouh

Pfizer Inc., Collegeville, PA, USA

M. R. Wilson · C. McDade

RTI Health Solutions, Research Triangle Park, NC, USA

M. J. Postma

Department of Pharmacy, University of Groningen, Groningen, The Netherlands

\section{J. Postma}

Research Institute of Science in Healthy Aging and healthcaRE (SHARE), University of Groningen, University Medical Center Groningen (UMCG), Groningen, The Netherlands

M.-C. Breton

Pfizer Canada Inc., Kirkland, QC, Canada effectiveness of a switch from the 13-valent (PCV13) to the 10-valent pneumococcal conjugate vaccine (PCV10) in Canada [1]. Our study estimated the incremental disease predicted to occur in Canada should there be a change in vaccination program from PCV13 to PCV10, using historical trends of individual serotypes contained in these vaccines. The model estimated that the switch could result in an additional 726,531 cases of disease over 10 years and that, despite a higher vaccine acquisition cost of PCV13, the net savings from lower disease incidence with continued PCV13 use would be $>\$ 500$ million.

A limitation of this predictive modeling was that scarce real-world evidence of such a switch from a higher to lower valent vaccine existed. Thus, a number of assumptions were included in the analysis to mitigate potential over- or underestimation of serotype dynamics. A critical assumption was that no changes in the serotypes that are unique to PCV13 (3, 6A, 19A) would be observed for at least 2 years after a vaccine policy change given the residual influence of many cohorts previously vaccinated with PCV13.

Since the publication of our model, surveillance data from Belgium have been published highlighting the real-world impact of a change from PCV13 to PCV10 [2]. Belgium introduced the 7-valent PCV (PCV7) in 2007 and subsequently replaced PCV7 with PCV13 in 2011. 
After near elimination of serotype 19A invasive pneumococcal disease (IPD) in Belgian children $\leq 2$ years of age ( 2 cases each were reported in 2015 and 2016), a switch to PCV10 was made in the two regional immunization programs in July 2015 (Flanders) and May 2016 (Wallonia). By the end of 2017, only 818 months following the switch, a nationwide ten-fold increase in serotype 19A IPD cases in children $\leq 2$ years of age was observed ( 21 cases reported). This increase continued in 2018, with ten serotype $19 \mathrm{~A}$ isolates detected in the first quarter of the year [2].

Acknowledging the complexities in interpreting these data and inherent difficulties in assessing causalities, these data could support our analysis and may suggest that our original assumptions and results could be conservative. In our model, assuming that re-emergence of previously covered serotypes could begin in children after only 1 year following the switch to PCV10 would result in an additional 134,122 cases of pneumococcal disease over 10 years compared with the original analysis (Table 1). This would translate to an additional $\$ 57 \mathrm{mil}-$ lion in costs to the Canadian health system because of fewer cases of disease.

While the reemergence of serotype 19A in Belgium was rapid and statistically significant, longer follow-up is necessary to determine whether these trends continue and to better understand potential causal relation with the switch to PCV10. Analyzing changes in IPD in non-vaccinated age groups (those not directly influenced by the switch) is crucial to understanding the impact of both vaccines on herd effect. This in turn can inform re-analyses of predictive models that will determine the true cost and effectiveness of changing PCV program policies.

Taking the complexities of pneumococcal epidemiology as well as the diverse direct and indirect effects of vaccination into account, we acknowledge limitations in our transfer of Belgium findings to a completely different setting. As was noted in a recently published correspondence [2], there are limitations to these data given the small sample size in passive surveillance and short time horizon [3]. Few examples of countries that have switched from PCV13 to PCV10 exist; however, the only evidence of impact beyond Belgium is from Casablanca, Morocco, where in a single hospital 4 years after sequential PCV13 then PCV10 implementation no change in serotype 19A across all years with PCV13 and PCV10 was observed [4]. Ergo, our results should be considered with caution and be conceived as only indicative of potential developments in the years to come.

Table 1 Additional cases of pneumococcal disease in Canada with PCV10 vs. PCV13 over 10 years across all ages

\begin{tabular}{lrrr}
\hline & 2-Year lag $^{\mathbf{a}}$ & 1-Year lag & Difference \\
\hline Number of cases of: & & & \\
Pneumococcal bacteremia & 3009 & 3146 & 137 \\
Pneumococcal meningitis & 1609 & 1682 & 73 \\
Pneumococcal acute otitis media & 709,073 & 839,881 & 130,808 \\
Nonhospitalized pneumococcal pneumonia & 10,285 & 12,790 & 2505 \\
Hospitalized pneumococcal pneumonia & 38,556 & 39,154 & 598 \\
Total cases & 762,531 & 896,653 & 134,122 \\
Deaths & 4399 & 4404 & 5 \\
Total costs & $\$ 523,307,951$ & $\$ 580,401,137$ & $\$ 57,093,186$ \\
\hline
\end{tabular}

${ }^{a}$ Results from Wilson et al. [1] 


\section{ACKNOWLEDGEMENTS}

Funding. This work was sponsored by Pfizer, Inc. No article processing charges were received by the journal for the publication of this article. All authors had full access to all of the data in this study and take complete responsibility for the integrity of the data and accuracy of the data analysis.

Authorship. All named authors meet the International Committee of Medical Journal Editors (ICMJE) criteria for authorship for this article, take responsibility for the integrity of the work as a whole, and have given their approval for this version to be published.

Disclosures. This work was sponsored by Pfizer, Inc. Michele Wilson is an employee of RTI Health Solutions, who were paid consultants to Pfizer in connection with the development of this commentary. Cheryl McDade is an employee of RTI Health Solutions, who were paid consultants to Pfizer in connection with the development of this commentary. Maarten Postma was a paid consultant to Pfizer (or received an honorarium from Pfizer) in connection with the development of this commentary. Matt Wasserman is an employee of Pfizer, Inc., who provided funding for the development of this commentary. MarieClaude Breton is an employee of Pfizer, Inc., who provided funding for the development of this commentary. Heather Sings is an employee of Pfizer, Inc., who provided funding for the development of this commentary. Raymond Farkouh is an employee of Pfizer, Inc., who provided funding for the development of this commentary.

Compliance with Ethics Guidelines. This article is based on previously conducted studies and does not contain any studies with human participants or animals performed by any of the authors.

Peer Review. Please note, contrary to the journal's standard single-blind peer review process, as a commentary this article underwent review by a member of the journal's Editorial Board.

Open Access. This article is distributed under the terms of the Creative Commons Attribution-NonCommercial 4.0 International License (http://creativecommons.org/licenses/ by-nc/4.0/), which permits any noncommercial use, distribution, and reproduction in any medium, provided you give appropriate credit to the original author(s) and the source, provide a link to the Creative Commons license, and indicate if changes were made.

\section{REFERENCES}

1. Wilson M, Wasserman M, Jadavi T, Postma M, Breton M-C, Peloquin F, Earnshaw S, McDade C, Sings H, Farkouh R. Clinical and economic impact of a potential switch from 13-valent to 10-valent pneumococcal conjugate infant vaccination in Canada. Infect Dis Ther. 2018;7:353-71. https://doi.org/10.1007/s40121018-0206-1

2. Desmet S, Verhaegen J, Van Ranst M, Peetermans WE, Lagrou K. Switch in a childhood pneumococcal vaccination program from PCV13 to PCV10: a defendable approach? Lancet Infect Dis. 2018. https://doi.org/10.1016/S14733099(18)30346-3 (published online July 9).

3. Izurieta $P$, Breuer T. Interpretation of the switch in a childhood pneumococcal vaccination programme from PCV13 to PCV10 in Belgium. Lancet Infect Dis. 2018;18(8):831-2.

4. Diawara I, et al. Invasive pneumococcal disease among children younger than 5 years of age before and after introduction of pneumococcal conjugate vaccine in Casablanca, Morocco. Int J Infect Dis. 2015;40:95-101. 